\title{
Nonorthogonal PEEC Formulation for Time- and Frequency-Domain EM and Circuit Modeling
}

\author{
Albert E. Ruehli, Life Fellow, IEEE, Giulio Antonini, Joris Esch, Jonas Ekman, Anita Mayo, and \\ Antonio Orlandi, Senior Member, IEEE
}

\begin{abstract}
Electromagnetic solvers based on the partial element equivalent circuit (PEEC) approach have proven to be well suited for the solution of combined circuit and EM problems. The inclusion of all types of Spice circuit elements is possible. Due to this, the approach has been used in many different tools. Most of these solvers have been based on a rectangular or Manhattan representation of the geometries. In this paper, we systematically extend the PEEC formulation to nonorthogonal geometries since many practical EM problems require a more general formulation. Importantly, the model given in this paper is consistent with the classical PEEC model for rectangular geometries. Some examples illustrating the application of the approach are given for both the time and frequency domain.
\end{abstract}

Index Terms-Circuit modeling, partial element equivalent circuit (PEEC) method, full-wave analysis.

\section{INTRODUCTION}

$\mathbf{T}$ HE NEED for practical computational tools and techniques as well as models for realistic electromagnetic compatibility (EMC) and electrical interconnect and package (EIP) problems has increased drastically over the last few years with the faster speed of digital electronic chips and with the increased frequencies in today's RF circuits. In response to this progress in technologies, EM modeling techniques, and EM solvers have also made progress at an impressive pace. This has resulted in specialized versions of the modeling techniques. Solvers are tailored for a specific class of problems, which allows increased efficiency. An EM modeling approach must be classified as suitable for a particular application range e.g., electrical machines, scattering problems, waveguide analysis or, again, EMC or EIP problems. Even the EMC and the EIP classes can be further subdivided into subproblems for which a particular solution approach yields the best results [1]. In this paper, we consider the surface and volume integral equation based partial element equivalent circuit (PEEC) technique applied to heterogeneous combined circuit and EMC and/or EIP problems. Examples of other approaches for mixed EM-circuit problems are given for the transmission-line model (TLM) in [2] and for the finite-difference time-domain (FDTD) technique in [3].

Manuscript received July 2, 2002; revised December 2, 2002.

A. E. Ruehli, J. Esch, and A. Mayo are with the IBM Research Division, Yorktown Heights, NY 10598 USA (e-mail: ruehli@us.ibm.com).

G. Antonini and A. Orlandi are with the University of L'Aquila, L'Aquila 67040 AQ, Italy.

J. Ekman is with the Luleå University of Technology, SE-971 87 Luleå, Sweden.

Digital Object Identifier 10.1109/TEMC.2003.810804
The PEEC approach has evolved over the years with a focus toward EMI and EIP problems. In the beginning, full-wave solutions were not necessary for many aspects of the problems and the quasi static solutions were used. They are still in use, for capacitance [4]-[6] and inductance $\left(L_{p}\right)$ PEEC problems [7]-[12]. With the increase in the speed and frequency ranges of the very large-scale integration (VLSI) chips, higher frequency solutions became necessary. This made the use of more consistent models necessary, and many variants of PEEC models were devised for different applications [13]-[22]. A clear and easy notation has been devised to differentiate between the many different possible PEEC models. As an example, the notation $\left(L_{p}, P_{n}, R, \tau\right)$ PEEC means that the model includes partial inductance $L_{p}$, normalized coefficients of potential $P_{n}$, resistance $R$, and delays $\tau$. For a specific application, other combinations of elements may be more suitable. The delays in time are equivalent to the retardation, in the frequency domain, as is apparent from the Laplace transform where $\mathcal{L} f(t-\tau)=F(s) e^{-s \tau}$. The PEEC method can be applied in both the TD and the frequency domain, very much like a typical Spice-type circuit solver where the option . ac leads to a frequency-domain analysis while tran corresponds to a TD analysis. TD models are used extensively for modeling VLSI circuits and chips while frequency-domain models are used for RF type applications.

In this paper, we focus on a systematic extension of the PEEC solution for nonorthogonal geometries in both the time and the frequency domain. Early on in the history of PEEC models, simplified nonorthogonal geometries where modeled in terms of rectangular bars with arbitrary orientations [9], [23], [11], [12]. Very recently, new approaches have been presented for nonorthogonal PEEC models using triangular cells with other approaches e.g., [24], [25], [21]. In this paper, we further develop the quadrilateral or hexahedral formulation presented in [26], [27]. The quadrilateral and hexahedral shapes are used to represent the different conductors as well as dielectric regions. Quadrilateral surface cells have been used successfully for EM modeling using the integral equation solution approach e.g., [28], [29].

Importantly, the new, general formulation retains all the properties of the orthogonal PEEC method. In fact, it is intriguing that the topology of the resultant general PEEC circuit model is exactly the same for rectangular and nonorthogonal geometries provided that triangular cells are not used [30]. For example, triangular surface cells need three basis functions rather than two as is the case for rectangular as well as quadrilateral surface cells. Hence, the new approach in this paper retains the flexibility of the conventional orthogonal PEEC approach, and 
importantly, properties like full wave and full spectrum. The full-wave aspects refer to the fact that up to a high-frequency limit, all modes of propagation are calculated. The full-spectrum label means that the method does not have a low frequency limit as is the case for many other EM methods. The PEEC solution is valid down to and including a $d c$ solution which is very important for the modeling of TD problems. This is in part why the full-wave model is practically the same as the quasistatic model with the exception of the delays or retardation provided that the appropriate circuit elements are included in the model. Further, the PEEC model has been extended to accurately include models for dielectrics [13], [22] and also scattering or incident fields [31]. The nonorthogonal extension in this paper can be applied to all aspects of modeling like $\left(L_{p}, R\right)$ PEEC inductance models or other reduced models, besides the full wave $\left(L_{p}, P_{n}, R, \tau\right)$ PEEC models. Example applications for the nonorthogonal approach are arbitrary shaped printed circuit antennas or the modeling of connectors including their EMI radiation. Other EMC and EIP applications point to the strength of the method. For example, the method has been applied for the modeling of high-voltage towers [15], and the analysis of printed circuit boards, e.g., [16], [26], [22]. Also, comparisons with measurement and the solution with other techniques have been made [32], [33].

Section II gives the derivation of the nonorthogonal PEEC model. A short introduction on the evaluation of the PEEC circuit elements is given in Section III. General aspects of the circuit solver were considered in Section IV. Finally, results from numerical experiments are given in Section V.

\section{NONORTHOGONAL PEEC MODEL}

\section{A. Nonorthogonal Formulation}

In this section, we give an outline of the geometrical nonorthogonal extension to the PEEC method. The objects can be quadrilateral and/or hexahedral conductors or dielectrics which can be both orthogonal and/or nonorthogonal.

The formulation utilizes a global as well as a local coordinate system. The key global coordinate system uses conventional orthogonal coordinates $x, y, z$ where a global vector $\boldsymbol{F}$ is of the form $\boldsymbol{F}=F_{x} \hat{x}+F_{y} \hat{\boldsymbol{y}}+F_{z} \hat{z}$. Therefore, the global unit vectors $\hat{\boldsymbol{x}}, \hat{\boldsymbol{y}}$, and $\hat{z}$ are position independent. We mark a vector in the global coordinates as $\boldsymbol{r}_{\boldsymbol{g}}$ for a clear distinction from the local coordinates. The local coordinates $a, b, c$ are used to separately represent each specific possibly nonorthogonal object. We call the unit vectors $\hat{\boldsymbol{a}}, \hat{\boldsymbol{b}}, \hat{\boldsymbol{c}}$ so that they are not confused with circuit variables like voltage $v$ etc. Details for nonorthogonal coordinate systems can be found in several texts, e.g., [34]. First, we very briefly introduce the local coordinate system we use for a hexahedral element shown in Fig. 1. The purpose of the local coordinates is to identify the location of any point belonging to the hexahedron in terms of the variables $a, b, c$ where $a \in[-1,+1]$ and where $a$ can mean $a=a, b, c$. The purpose of all this is to uniquely map a point $a, b, c$ into a point in the global coordinates $\boldsymbol{r}_{\boldsymbol{g}}$. We accomplish this by specifying eight vectors $\boldsymbol{r}_{\boldsymbol{g}_{\boldsymbol{k}}}$ where $k=0,7$ with the coordinates $x_{k}, y_{k}, z_{k}$. It will be clear that the corners of the hexahedron are reached when $a, b, c= \pm 1$.

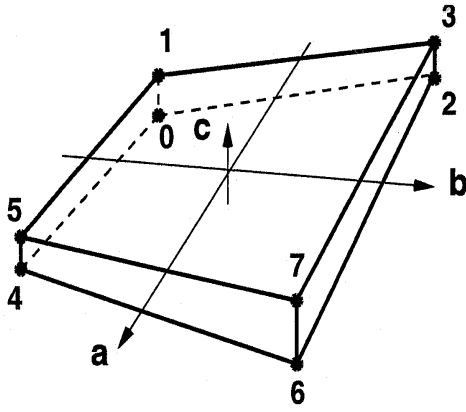

Fig. 1. Basic hexahedral element or object with local coordinates.

Next, we create clean assignment of the corner indices in Fig. 1, for the hexahedron using a binary code for the symbols $a, b, c$, where the -1 coordinates map into logical zeros, and +1 coordinates map into logical ones. The order of the logical variables is $a b c$. Hence, for example, the binary code $a b c=011$ corresponds to the corner $a=0, b=1$, and $c=1$ and its decimal equivalent is 3 for corner 3 as can be verified in Fig. 1 . This makes the assignment unique and easy to remember. As indicated above, all local coordinates have to relate back to the global $x, y, z$ coordinates. Therefore, a unique representation is needed for the mapping from a local point $a, b, c$ on an object to the global point $\boldsymbol{r}_{\boldsymbol{g}}$. Mapping a point in the above hexahedron from a local coordinate point $a, b, c$ into a global coordinate point $x, y, z$ is described by

$$
x=\sum_{k=0}^{7} N_{k}(a, b, c) x_{k}
$$

which is applied for $x=x, y, z$. The coefficients in (2.1) are given by

$$
\begin{aligned}
& N_{0}=1 / 8(1-a)(1-b)(1-c) \\
& N_{1}=1 / 8(1-a)(1-b)(1+c) \\
& N_{2}=1 / 8(1-a)(1+b)(1-c) \\
& N_{3}=1 / 8(1-a)(1+b)(1+c) \\
& N_{4}=1 / 8(1+a)(1-b)(1-c) \\
& N_{5}=1 / 8(1+a)(1-b)(1+c) \\
& N_{6}=1 / 8(1+a)(1+b)(1-c) \\
& N_{7}=1 / 8(1+a)(1+b)(1+c)
\end{aligned}
$$

where $a \in[-1,+1]$ and again $a=a, b, c$. The close relation to the binary variables is evident in (2.2).

With this, we are in a position to also express the tangential vectors with respect to the local coordinates as

$$
\frac{\partial \boldsymbol{r}_{\boldsymbol{g}}}{\partial a}=\frac{\partial x}{\partial a} \hat{\boldsymbol{x}}+\frac{\partial y}{\partial a} \hat{\boldsymbol{y}}+\frac{\partial z}{\partial a} \hat{z}
$$

where the derivatives are found from (2.1) and (2.2). Finally, the magnitude of the tangential vector $h_{a}=\left|\partial \boldsymbol{r}_{\boldsymbol{g}} / \partial a\right|$ where the position dependent unit vectors can be determined from $\hat{\boldsymbol{a}}=$ $\left(\partial \boldsymbol{r}_{\boldsymbol{g}} / \partial a\right) / h_{a}$ where again $a=a, b, c$. With this we are prepared to formulate the geometrical aspects for the nonorthogonal PEEC circuit elements. 
In the PEEC circuit solution, terminal or nodal variables are associated with each of the resultant circuit elements will be collected in an overall circuit solver vector of unknowns. The solution vector variables are quantities like the potentials $\mathbf{\Phi}$, and other conventional circuit variables like $\boldsymbol{i}, \boldsymbol{v}, \boldsymbol{q}$ where $\boldsymbol{i}$ is the currents, $\boldsymbol{v}$ the voltage, and $\boldsymbol{q}$ some charges, etc. If no other circuit elements are included, the solution vector used is $(\boldsymbol{\Phi}, \boldsymbol{i})^{T}$ as the only unknowns since this vector directly yields the most useful EM circuit output variables. Here we point out that in part, the inclusion of both $\Phi$, and $i$ is, responsible for the fullspectrum property of the solution methodology such that the correct $d c$ solution is obtained.

\section{B. Basic Integral Equations for Nonorthogonal Geometries}

The PEEC formulation uses an integral equation solution of Maxwell's equations based on the total electric field. An integral or inner product is used to reformulate each term of (2.10) into the circuit equations. This inner product integration converts each term into the fundamental form $\int \boldsymbol{E} \cdot d l=V$ where $V$ is a voltage or potential difference across the circuit element. It is evident that this transforms the sum of the electric fields in (2.4) into the Kirchhoff voltage law (KVL).

The starting point is the total electric field at or in the material which is

$$
\boldsymbol{E}^{i}\left(\boldsymbol{r}_{\boldsymbol{g}}, t\right)=\frac{\boldsymbol{J}\left(\boldsymbol{r}_{\boldsymbol{g}}, t\right)}{\sigma}+\frac{\partial \boldsymbol{A}\left(\boldsymbol{r}_{\boldsymbol{g}}, t\right)}{\partial t}+\nabla \phi(\boldsymbol{r g}, t)
$$

where $\boldsymbol{E}^{i}$ is the incident electric field, $\boldsymbol{J}$ is the current density in a conductor and $A$ and $\phi$ are vector and scalar potentials, respectively. As indicated above, the dielectric areas are taken into account as an excess current rather than a capacitance with the scalar potential using the volume equivalence theorem [35]. This is accomplished by adding and subtracting $\epsilon_{0} \partial \boldsymbol{E} / \partial t$ at in the Maxwell equation for $\boldsymbol{H}$, or

$$
\begin{aligned}
\nabla \times \boldsymbol{H}\left(\boldsymbol{r}_{\boldsymbol{g}}, t\right)=\boldsymbol{J}_{C}\left(\boldsymbol{r}_{\boldsymbol{g}}, t\right) & +\epsilon_{0}\left(\epsilon_{r}-1\right) \\
& \times \frac{\partial \boldsymbol{E}\left(\boldsymbol{r}_{\boldsymbol{g}}, t\right)}{\partial t}+\epsilon_{0} \frac{\partial \boldsymbol{E}\left(\boldsymbol{r}_{\boldsymbol{g}}, t\right)}{\partial t} .
\end{aligned}
$$

Here, the current in (2.5) is written as a total current

$$
\boldsymbol{J}\left(\boldsymbol{r}_{\boldsymbol{g}}, t\right)=\boldsymbol{J}_{C}\left(\boldsymbol{r}_{\boldsymbol{g}}, t\right)+\epsilon_{0}\left(\epsilon_{r}-1\right) \frac{\partial \boldsymbol{E}\left(\boldsymbol{r}_{\boldsymbol{g}}, t\right)}{\partial t}
$$

where $\boldsymbol{J}_{C}$ is the conductor current and the remainder of the equations is the equivalent polarization current due to the dielectric.

The vector potential $A$ is for a single conductor at the field point $\boldsymbol{r}_{\boldsymbol{g}}$ given by

$$
\boldsymbol{A}\left(\boldsymbol{r}_{\boldsymbol{g}}, t\right)=\mu \int_{v^{\prime}} G\left(\boldsymbol{r}_{\boldsymbol{g}}, \boldsymbol{r}_{\boldsymbol{g}^{\prime}}\right) \boldsymbol{J}\left(\boldsymbol{r}^{\prime}, t_{d}\right) d v^{\prime}
$$

where the retardation time is given by $t_{d}=t-\left|\boldsymbol{r}_{\boldsymbol{g}}-\boldsymbol{r}_{\boldsymbol{g}^{\prime}}\right| / c$ which simply is the free-space travel time between the points $\boldsymbol{r}_{\boldsymbol{g}}$ and $\boldsymbol{r}_{\boldsymbol{g}^{\prime}}$. It is noted that in the formulation derived here, both the retardation and the Green's functions are free-space quantities where

$$
G\left(\boldsymbol{r}_{\boldsymbol{g}}, \boldsymbol{r}_{\boldsymbol{g}^{\prime}}\right):=\frac{1}{4 \pi} \frac{1}{\left|\boldsymbol{r}_{\boldsymbol{g}}-\boldsymbol{r}_{\boldsymbol{g}^{\prime}}\right|}
$$

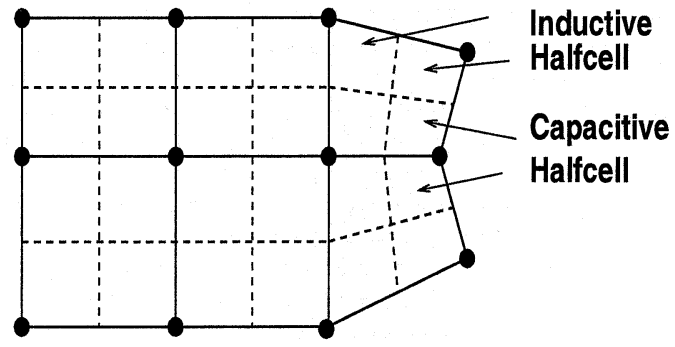

Fig. 2. Geometry with several quadrilateral elements.

The scalar potential is similarly

$$
\phi\left(\boldsymbol{r}_{\boldsymbol{g}}, t\right)=\frac{1}{\epsilon_{0}} \int_{v^{\prime}} G\left(\boldsymbol{r}_{\boldsymbol{g}}, \boldsymbol{r}_{\boldsymbol{g}^{\prime}}\right) q\left(\boldsymbol{r}_{\boldsymbol{g}^{\prime}}, d\right) d v^{\prime} .
$$

Finally, using the above, we can formulate an integral equation for the electric field at a point $\boldsymbol{r}_{\boldsymbol{g}}$ which is to be located either inside a conductor or inside a dielectric region. Starting from (2.4) with the externally applied electric field set to zero, and substituting for $A$ and $\phi$ from (2.7) and (2.9), respectively. For more details, please see any one of the PEEC papers, e.g., [36], [14], [20], [37]. A derivation of the PEEC model with the rectangular, finite dielectrics is given in [13]. The final integral equation to be solved is

$$
\begin{aligned}
& \hat{\boldsymbol{n}} \times \boldsymbol{E}^{i}\left(\boldsymbol{r}_{\boldsymbol{g}}, t\right) \\
&=\hat{\boldsymbol{n}} \times\left[\frac{\boldsymbol{J}\left(\boldsymbol{r}_{\boldsymbol{g}}, t\right)}{\sigma}\right] \\
&+\hat{\boldsymbol{n}} \times\left[\mu \int_{v^{\prime}} G\left(\boldsymbol{r}_{\boldsymbol{g}}, \boldsymbol{r}_{\boldsymbol{g}^{\prime}}\right) \frac{\partial \boldsymbol{J}\left(\boldsymbol{r}_{\boldsymbol{g}^{\prime}}, t_{d}\right)}{\partial t} d v^{\prime}\right] \\
&+\hat{\boldsymbol{n}} \times\left[\epsilon_{0}\left(\epsilon_{r}-1\right) \mu \int_{v^{\prime}} G\left(\boldsymbol{r}_{\boldsymbol{g}}, \boldsymbol{r}_{\boldsymbol{g}^{\prime}}\right) \frac{\partial^{2} \boldsymbol{E}\left(\boldsymbol{r}_{\boldsymbol{g}^{\prime}}, t_{d}\right)}{\partial t^{2}}\right] \\
&+\hat{\boldsymbol{n}} \times\left[\frac{\nabla}{\epsilon_{0}} \int_{v^{\prime}} G\left(\boldsymbol{r}_{\boldsymbol{g}}, \boldsymbol{r}_{\boldsymbol{g}^{\prime}}\right) q\left(\boldsymbol{r}_{\boldsymbol{g}^{\prime}}, t_{d}\right) d v^{\prime}\right]
\end{aligned}
$$

where $\hat{\boldsymbol{n}}$ is the surface normal to the body surfaces. Equation (2.10) is a TD formulation which can easily be converted to the frequency domain by using the Laplace transform operator $s=\partial / \partial t$ and where the time retardation will transform to $e^{-s \tau}$ where $\tau$ is the delay time.

\section{Discretization of Conductor and Dielectrics Geometries}

Section II-B shows the integral equation (2.10) which needs to be solved. In this section we determine the discrete equivalent and the circuit elements for nonorthogonal geometries. The basic hexahedral element or object is shown in Fig. 1 and an example for the connection between quadrilateral and orthogonal surface elements only is shown in Fig. 2. In PEEC we distinguish between inductive and capacitive cells and subdivisions where the inductive cells connect between the nodes and capacitive cells surround the nodes. The compatibility between quadrilateral and rectangular cells is apparent from Fig. 2. The bodies are joined together simply by joining the nodes where the adjoining half cells in Fig. 2 combine into full cells. To represent the current flow in orthogonal cells, we use the convenient weighting function $J=I /(W T)$ where $I, J$ are the current 
and current density and $W, T$ are the cell width and thickness, respectively. For the general case, we assume also that the conductor thickness is also subdivided into cells to take the thickness skin effect into account. This leads to the hexahedral elements in Fig. 1 for the nonorthogonal case. Since PEEC is an integral equation based formulation the usual volume-filament (VFI) skin effect models result automatically from the subdivision of the interiors of the conductors [36]. The generalization of the current distribution for nonorthogonal hexahedral cell shapes is given by

$$
J_{a}=\frac{h_{a}}{\left|\frac{\partial \boldsymbol{r}_{\boldsymbol{g}}}{\partial a} \cdot\left(\frac{\partial \boldsymbol{r}_{\boldsymbol{g}}}{\partial b} \times \frac{\partial \boldsymbol{r}_{\boldsymbol{g}}}{\partial c}\right)\right|} I_{a}
$$

where $J_{b}$ and $J_{c}$ can easily be found by permuting the indices. We call the quotient in (2.11) the weight $w_{a}$ which simplifies (2.11) to $J_{a}=w_{a} I_{a}$. We should note that all the above quantities are a function of the local position coordinates $a, b, c$. Next, we use an integral or inner product operator

$$
V_{a}=\int_{a} \int_{b} \int_{c} w_{a} \hat{\boldsymbol{a}} \cdot \boldsymbol{E}\left|\frac{\partial \boldsymbol{r}_{\boldsymbol{g}}}{\partial a} \cdot\left(\frac{\partial \boldsymbol{r}_{\boldsymbol{g}}}{\partial b} \times \frac{\partial \boldsymbol{r}_{\boldsymbol{g}}}{\partial c}\right)\right| d a d b d c
$$

to integrate the terms of (2.10) where $\boldsymbol{E}(a, b, c)$ is the $\boldsymbol{E}$ field term to be integrated. We need to apply the inner product to each term in (2.10) to transform each term to a voltage drop across a circuit element in the KVL equation. Next, we integrate the right-hand terms of the integral equation (2.10). After applying the inner product, the first element on the right-hand side leads to the series resistance term in the form

$$
R_{a}=\frac{1}{\sigma} \int_{a} \int_{b} \int_{c} \frac{h_{a}^{2}}{\left|\frac{\partial \boldsymbol{r}_{\boldsymbol{g}}}{\partial a} \cdot\left(\frac{\partial \boldsymbol{r}_{\boldsymbol{g}}}{\partial b} \times \frac{\partial \boldsymbol{r}_{\boldsymbol{g}}}{\partial c}\right)\right|} d a d b d c .
$$

The second right-hand side term of (2.10), after applying (2.12) is a generalization of the partial inductance concept for nonorthogonal problems, or

$$
\begin{aligned}
L p a_{a a^{\prime}}= & \mu \int_{a} \int_{b} \int_{c} \int_{a^{\prime}} \int_{b^{\prime}} \int_{c^{\prime}}\left(\hat{\boldsymbol{a}} \cdot \hat{\boldsymbol{a}}^{\prime}\right) h_{a^{\prime}} \\
& \cdot G\left[\boldsymbol{r}_{\boldsymbol{g}}(a, b, c) ; \boldsymbol{r}_{\boldsymbol{g}}\left(a^{\prime}, b^{\prime}, c^{\prime}\right)\right] d a^{\prime} d b^{\prime} d c^{\prime} h_{a} d_{a} d_{b} d_{c} .
\end{aligned}
$$

The charge density is in general of a similar form as (2.11). Of course, charge does not have a directional dependence, and the volume charge $q_{v}$ is given by

$$
q_{v}\left(a^{\prime}, b^{\prime}, c^{\prime}\right)=\frac{1}{\left|\frac{\partial \boldsymbol{r}_{\boldsymbol{g}}}{\partial a} \cdot\left(\frac{\partial \boldsymbol{r}_{\boldsymbol{g}}}{\partial b} \times \frac{\partial \boldsymbol{r}_{\boldsymbol{g}}}{\partial c}\right)\right|} Q
$$

where $Q$ is the total charge in the volume cell. For the conventional conductors, the charge will be restricted to the surface cells only. The gradient in (2.4) in the $\hat{\boldsymbol{a}}$ direction evaluates to

$$
\left.\nabla \phi\right|_{a}=\frac{1}{h_{a}} \frac{\partial \phi}{\partial a} .
$$

To maintain symmetry for the circuit elements or coefficients we approximate the derivative, with an integrated average over

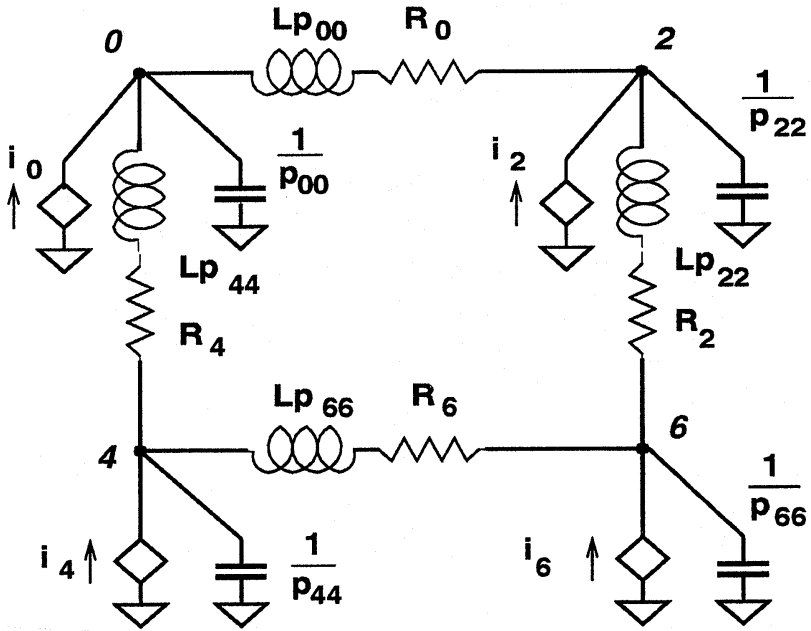

Fig. 3. PEEC model for top part of Fig. 1.

the two corner cells corresponding to the derivative where the spacing is the projection in the $\hat{\boldsymbol{a}}$ direction for the center to center distance $d_{a}$. This approximation implies that (2.16) simplifies to

$$
\left.\nabla \Phi\right|_{a} \approx \frac{1}{h_{a} d_{a}} \int \phi(a, b, c) d S_{l}
$$

where $S_{l}$ is a surface in the appropriate surface direction(s). Finally, by inserting for the potential $\phi$ in (2.17) and by cancelling the appropriate terms, we get for the normalized coefficients of potential

$$
\begin{aligned}
& P n_{a a^{\prime}}=\frac{1}{\epsilon} \int_{a} \int_{b} \int_{a^{\prime}} \int_{b^{\prime}} \\
& \cdot G\left[\boldsymbol{r}_{\boldsymbol{g}}(a, b, c) ; \boldsymbol{r}_{\boldsymbol{g}^{\prime}}\left(a^{\prime}, b^{\prime}, c^{\prime}\right)\right] d_{a}^{\prime} d_{b}^{\prime} d_{a} d_{b} .
\end{aligned}
$$

Here, $a, b, a^{\prime}, b^{\prime}$ are interpreted in a general sense for the appropriate cell orientations. The PEEC circuit for a quadrilateral element is shown in Fig. 3. It consists of four KVL loops. Specifically, a KVL loop involves two nodes with the capacitances to infinity and a partial inductance in series with a resistor. Hence, the element shown for the bottom layer only of the element shown in Fig. 1 consists of four loops to infinity where the PEEC topology for the orthogonal and the nonorthogonal case are the same, with the exception of the circuit element values. Importantly, the same modified nodal analysis (MNA) circuit solver can be used for both separately as well as the mixed cases.

Similar to the orthogonal case, the dielectrics are represented with additional circuit elements. We define the excess capacitance of a dielectric cell $\gamma$ as

$$
C_{\gamma}^{+}:=\epsilon_{0}\left(\epsilon_{\gamma}-1\right) / \int_{a} \int_{b} \int_{c} \frac{h_{a}^{2}}{\left|\frac{\partial \boldsymbol{r}_{\boldsymbol{g}}}{\partial a} \cdot\left(\frac{\partial \boldsymbol{r}_{\boldsymbol{g}}}{\partial b} \times \frac{\partial \boldsymbol{r}_{\boldsymbol{g}}}{\partial c}\right)\right|} d a d b d c
$$

where $\epsilon_{\gamma}$ is the dielectric constant of the dielectric cell. The equivalent circuit for the dielectric cell $\gamma$ is given by a partial inductance $L p_{\gamma \gamma}$ in series to a capacitor $C_{\gamma}^{+}$with the current $i=C_{\gamma}^{+}\left(d\left(\Phi_{a}-\Phi_{b}\right) / d t\right.$ where $\phi_{a}$ and $\phi_{b}$ are the potential at the two ends of the dielectric cell. This model for finite dielectrics is a very important part of the PEEC approach [38]. 


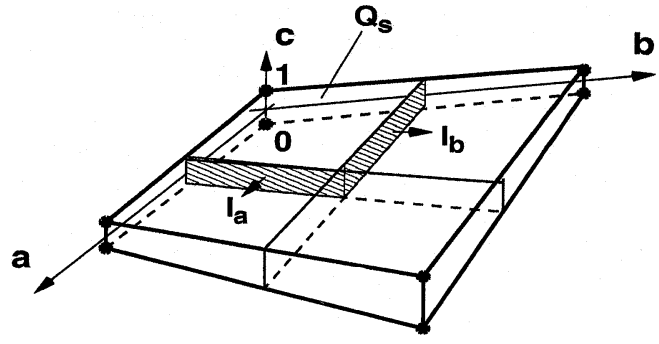

Fig. 4. Corner of cell for continuity equation.

\section{Continuity Equation and Kirchhoff's Current Law}

It is important that the continuity equation is satisfied at the cell level for the currents and charges. Its differential form is given by $\nabla \cdot \boldsymbol{J}+(\partial q / \partial t)=0$ where $\boldsymbol{J}$ is the current density and $q$ is the surface charge density. The continuity equation is applied at the location of each node. For this reason, we show the geometry of a corner in Fig. 4, corresponding to nodes 0 and 1 in Fig. 1. Since only one quarter of the elements surrounding the node is shown, we assume that the surface element in Fig. 4 may be connected to other similar surfaces along the $a-c$ and the $b-c$ surfaces. Hence, the volume for which the continuity equation is applied consists of the corners which are involved in the geometry surrounding the node(s). It is sufficient to consider only the corner elements, by ignoring the internal surfaces shown in Fig. 4, for simplicity.

Integrating the continuity equation over the corner yields

$$
\int_{v} \nabla \cdot \boldsymbol{J} d v=-\frac{\partial}{\partial t} \int_{v} q d v=\int_{s} \boldsymbol{J} \cdot \hat{\boldsymbol{n}} d S
$$

where the divergence theorem [39] is used in the last step and where the vector $\hat{\boldsymbol{n}}$ is normal to the surface $s$. The volume integral part pertains to the top and bottom $a-b$ surfaces corners connected to nodes 1 and 0 which are charged as indicated with $Q_{s}$ in Fig. 4. If the nodes 0 and 1 are shorted, then the charge density consists of two $\delta$-functions at the surfaces of the conductors with the surface charge $q(a, b, c)$ and the contributions at $c= \pm 1$ are

$$
Q=\int_{a=-1}^{0} \int_{b=-1}^{0} q(a, b)\left|\frac{\partial \boldsymbol{r}_{\boldsymbol{g}}}{\partial a} \times \frac{\partial \boldsymbol{r}_{\boldsymbol{g}}}{\partial b}\right| d a d b
$$

and where the surface charge can be found from (2.15) as $q_{ \pm}(a, b)=Q_{ \pm} /\left|\left(\partial \boldsymbol{r}_{\boldsymbol{g}} / \partial a\right) \times\left(\partial \boldsymbol{r}_{\boldsymbol{g}} / \partial b\right)\right|$. Inserting this into (2.21) yields the charges $Q_{ \pm}$on the corner surfaces.

Similarly, the currents associated with the corner nodes 0,1 are flowing through the cross-sectional areas indicted by $I_{a}$ in the $a$ direction and $I_{b}$ in the $b$ direction. The current through the section of conductor cross section for $I_{a}$ is given by

$$
I_{a}=\int_{b=-1}^{0} \int_{c=-1}^{0} I_{a} w_{a} \hat{\boldsymbol{a}} \cdot\left|\frac{\partial \boldsymbol{r}_{\boldsymbol{g}}}{\partial b} \times \frac{\partial \boldsymbol{r}_{\boldsymbol{g}}}{\partial c}\right| d b d c .
$$

Again, the same relationship holds for the equation for the $b$ coordinate. Adding all terms pertaining to the continuity equation we get, for one corner only

$$
\frac{d Q_{+}}{d t}+\frac{d Q_{-}}{d t}+I_{a}+I_{b}+=0
$$

which can be recognized as Kirchhoff's current law (KCL). It is evident that the continuity equation is satisfied since the admittance part of the MNA formulation method [40] is based on summing up all the current contributions at a node. Another important observation is that for systems which include retardation all the parts which belong to a node are mostly instantaneous, while the retardation must be included for all parts outside the volume. Since the partial inductances extend from one node to a neighbor node, we need to subdivide the partial inductances into two half- $s$ were the part at the node is instantaneous and where the second part which belongs to a neighboring cell must include a retarded partial mutual inductance. It should be noted that this does not introduce additional unknowns and it improves the TD stability [41].

\section{Evaluation OF CiRCUIT ElEMENTS}

One of the challenging subjects for the PEEC method is the evaluation of the circuit elements due to the high-accuracy requirements for the dense structures which must be solved for EMI and EIP problems. This issue is worst for elements corresponding to cells which coincide or are in close physical proximity. Unfortunately, the early work in this field, like inductance calculations in power systems, had different less dense applications where more approximate results were acceptable [42]. For speed and accuracy reasons, we utilize a multifunction approximation approach for the coefficient evaluation which is based on analytical results as well as on numerical solutions. Further, the PEEC method poses another challenge for the coefficient evaluation due to the large aspect ratios in the size of the conductors as will be shown below in an example in Section V. The evaluation of the partial inductances (2.14) for rectangular geometries has been the topic of many papers over the years e.g., [7], [9], [12], [43]. Further, the normalized coefficients of potential (2.18) are available in closed form for all rectangular cells in [4] and for parallel rectangular cells in [44].

The evaluation of the coefficients for the nonorthogonal case is even more challenging. The zero thickness self term as well as coefficients which are in a plane have been considered in a recent paper [45]. In order to evaluate these coefficients for the general nonorthogonal case we use a combination of analytic integration and Gaussian quadrature. Specifically, for physically close cells, we first divide each hexahedral cell into a set of layers in the $\mathrm{c}$ direction. The thickness of these layers is not uniform, but is determined by a compound Gaussian quadrature rule. Specifically, in the local coordinates the $k$ th layer is $a \in$ $[-1,+1], b \in[-1,+1], c \in\left[c_{k-1}, c_{k}\right]$. The values of the $\left\{c_{k}\right\}$ are the values of the nodes in the four-point Gaussian quadrature rule with $l v l$ levels of compounding. There are thus a total of $5(l v l)-1$ layers in each inductive cell. The value of $l v l$ depends on the aspect ratios of the cell and the accuracy required.

Once each cell has been subdivided, then the evaluation of the integral (2.14) is reduced to the evaluation $n z$ fourfold integrals of the form (3.24)

$$
\begin{aligned}
L^{s} p_{a a^{\prime}}= & \mu \int_{a} \int_{b} \int_{a^{\prime}} \int_{b^{\prime}}\left(\hat{\boldsymbol{a}} \cdot \hat{\boldsymbol{a}}^{\prime}\right) h_{a^{\prime}} \\
& \cdot G\left[\boldsymbol{r}_{\boldsymbol{g}}(a, b, c) ; \boldsymbol{r}_{\boldsymbol{g}}\left(a^{\prime}, b^{\prime}, c^{\prime}\right)\right] d a^{\prime} d b^{\prime} h_{a} d a d b
\end{aligned}
$$


where $n z$ is the product of the number of layers in each cell. Each fourfold integral is the integral over two quadrilaterals. We evaluate these integrals by subdividing each quadrilateral into uniform rectangles in the local $a$ and $b$ directions. Assuming that we integrate a rectangular domain $R=a \in[-1,+1], b \in$ $[-1,+1]$ into $n m$ subquadrilaterals $R_{i j}=(i-1) / n \leq a \leq$ $i / n,(j-i) / m \leq b \leq j / m$. The subdivisions $n$ and $m$ are chosen to maintain a reasonable aspect ratio as well as the required accuracy. The evaluation of the partial self- and near-mutual inductances (2.14) is more difficult than the normalized potential coefficients due to inner product. For this, we subdivide each rectangular domain into two triangles for which an analytical formulation exists. On each triangle we approximate $\hat{\boldsymbol{a}} \cdot \hat{\boldsymbol{a}}^{\prime} h_{a} h_{a^{\prime}}$, by a linear function which agrees with its vales at the three vertices of the triangle. Importantly, an analytical formula for the product of a linear function and the Green's function exists for a triangle [46]. We perform the outer integration in (2.14) with respect to $a, b$, and $c$ by using a nine-point sixth-order accurate product Gaussian quadrature rule. Once we have obtained an approximation $S_{1}$ to the integral (2.14) using this method, we divide each cell into twice as many $c$ layers as before, that is we double $l v l$, and repeat the procedure, obtaining another approximation, $S_{2}$. Then, we use second-order Richardson extrapolation to obtain our final estimate $S=\left(4 S_{2}-S_{1}\right) / 3$.

\section{General Circuit Solver Aspects}

In this section, we consider a general-purpose TD and frequency-domain solver implementation for the PEEC approach. As mentioned in Section II, the full spectrum property in PEEC is obtained by using a combined solution vector $(\boldsymbol{\Phi}, i)^{T}$ in the TD or frequency domain. This is based on the MNA circuit formulation [40] where $\boldsymbol{\Phi}$ is the potential and $\boldsymbol{i}$ are the cell and some branch currents. The zero potential $\phi=0$ corresponds to the ground node 0 or node at an infinite distance in space. We found that the inclusion of the potentials as unknowns has proven to be very valuable as output quantities. For example, for PC board plane to plane voltages [33] are simply given by the potential difference between the closely located planes. Alternatively, if charges are desired $\boldsymbol{q}$ is required for some elements instead of $\boldsymbol{\Phi}$ then they can be included in the MNA as part of the solution vector, e.g., Singhal and Vlach in [47]. Many practical problems consist of different Spice-type lumped circuit elements in addition to the PEEC model. These elements can easily be included in the circuit matrix using the usual MNA stamps [40], [48], and [49] for both the time and frequency domain. These matrix stamps have been developed for a multitude of circuit elements. A PEEC computer program may consist of a model element generator and several circuit solvers for the time and the frequency domain. The elements of the equivalent circuits generated by the PEEC discretization are stamped into the MNA circuit matrix and then the resultant circuit is solved using a sparse matrix code. Over the years, other loop based approaches like the MLA [50] have been applied to the PEEC problems. Mixed-circuit formulations including loop or mesh formulations have also been used since they can lead to efficient solution for some specialized problems [51], [52], [17]. One of the present research topics is the speed up of the solu- tion process. Non retarded $\left(L_{p}, P_{n}, R\right) P E E C$ and full wave solutions have been attempted using the model order reduction (MOR) approaches for circuit which do not have a multitude of input/output connections and a moderate number of relevant eigenvalues, e.g., [53]. This research is ongoing especially in the area of $\left(L_{p}, R\right) P E E C$ models for on chip applications where $L_{p}^{-1}$ is used to limit the radius of coupling for the partial mutual inductances, e.g., [43].

Much progress has been made very recently in the stable time solution of integral equation based full wave solutions. Early work, where mostly explicit numerical integration methods are employed is summarized in [54]. Since then, several researchers have shown that much more stable solutions can be obtained with implicit numerical integration methods, e.g., [55], [14], [56], [57]. This implies that today, the stable transient analysis of large structures can be accomplished efficiently. It should be noted that the full-spectrum property is a very important aspect of the method for both the time and the frequency domain where the input spectrum often ranges from do to the maximum permissible frequency content.

The Spice input language is an other aspect of an EM circuit solver which makes its use practical and easier to understand. The utility of this was well recognized early on and the Spice syntax has been used in all the "Ciao" series of PEEC solvers, e.g., [14]. We call the code based on the formulation in this paper IBMciao. We continued to enhance the language to include the description of the nonorthogonal conductors. This approach also allows a rapid combined TD and frequency-domain analysis which lead to a better understanding of the electrical and electromagnetic behavior of the systems, and helps the designer to gain insight into the way the various parameters of the system impact its overall performance. While the majority of the language used for the description of the various types of circuit elements is based, as much as possible, on the conventional Spice language, some specialized syntax is used in company specific versions (e.g., Motorola's Mspice and IBM's PowerSpice) and commercial versions like PSpice and Hspice. One of the recommended enhancements in the basic Spice language is an improved syntax for mutual inductances

\section{Kname Lname1 Lname2 <type> number < delay $>$.}

The new proposed option assigns directly the value of the mutual inductance rather than the transformer coupling factor $k=L 12 / \sqrt{L 11 L 22}$ which is computationally expensive. More specifically, the default option could be that the coupling factor is assigned (in which case the assignment " $k$ " for "type" is optional), while for the case where the more desirable mutual inductance value is explicitly given the assignment " $m$ " for "type" is required. The savings are evident for circuits with many mutual inductance like PEEC circuits. Also, the delay between the inductors allows the inclusion of retarded PEEC models in Spice [20].

\section{NUMERICAL EXPERIMENTS}

We give several examples in this paper to illustrate the versatility of the approach. As a first example, we model a dipole 


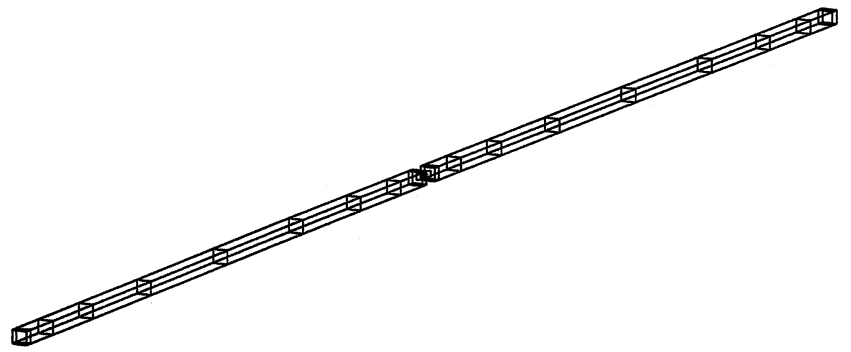

Fig. 5. Example 200-mm-thin dipole antenna.

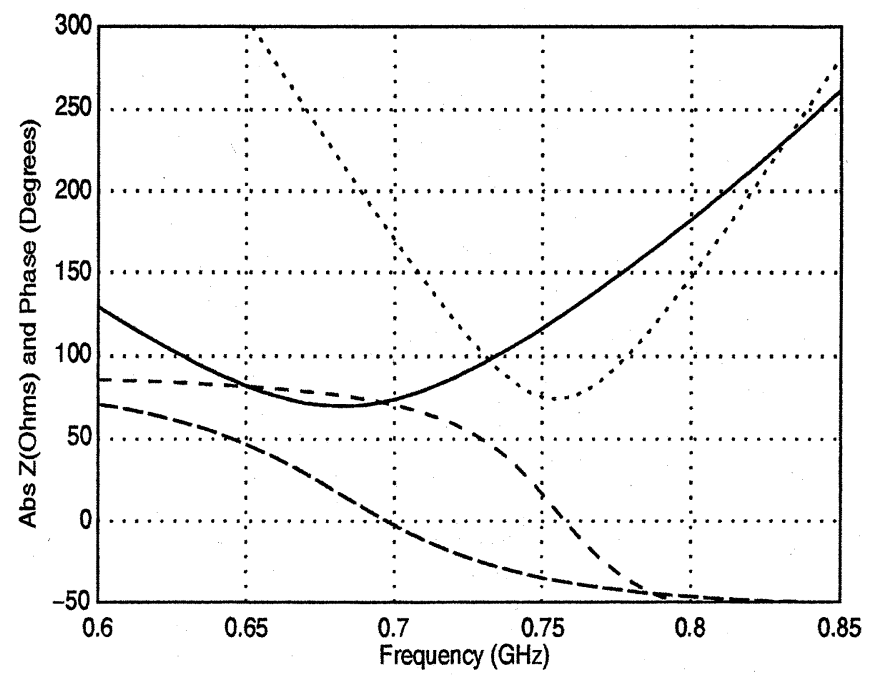

Fig. 6. Input impedance and phase for thin $1 \times 1 \mathrm{~mm}$, and $1 \times 1-\mu \mathrm{m}$ lossless dipole.

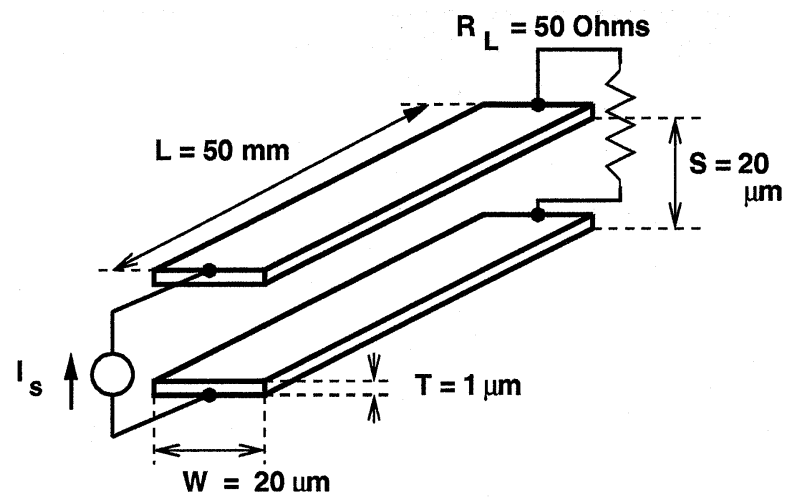

Fig. 7. Lossy TL example problem.

shown in Fig. 5, with a length of $200 \mathrm{~mm}$ to compare with a well known results and to confirm the accuracy of the solution. Since the theoretical results are available for the lossless case we use a zero resistance $\left(L_{p}, P_{n}, \tau\right)$ PEEC model. The meshing we use is nonuniform with smaller cells toward the center gap as well as the ends of the dipole. At the center, we introduced a gap of $2 \mathrm{~mm}$. Results are given for two dipoles with different cross sections to show that in order to obtain a close agreement with the theoretical value, we had to resort to a very small $1 \mu \mathrm{m} \times 1 \mu \mathrm{m}$ cross section. Our results are shown in Fig. 6. The magnitude of the impedance is $73.5 \Omega$ at resonance, which agrees well with the theoretical value of $73.1 \Omega$. The resonance frequency for this

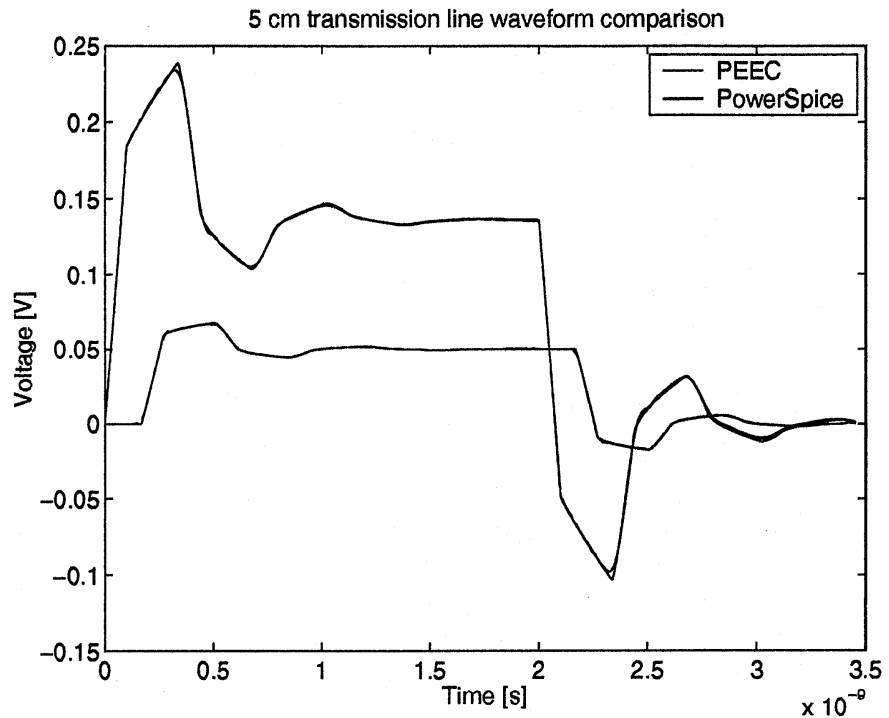

Fig. 8. Waveform comparisons for 3-D PEEC and 2-D TL solvers.
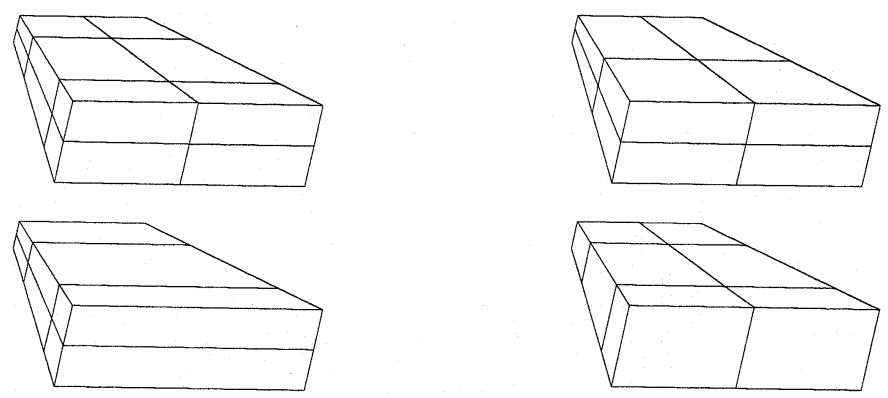

Fig. 9. Capacitive and inductive-resistive cells for a single nonorthogonal hexahedron. Upper-left: Capacitive surface cells; Lower-left: Inductive volume cells $b$-directed current (right); Upper-right: Inductive volume cells $a$-directed current (front); Lower-right: Inductive volume cells $c$-directed current (up).

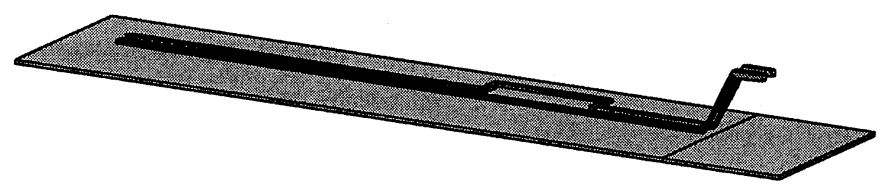

Fig. 10. TL with nonorthogonal geometry.

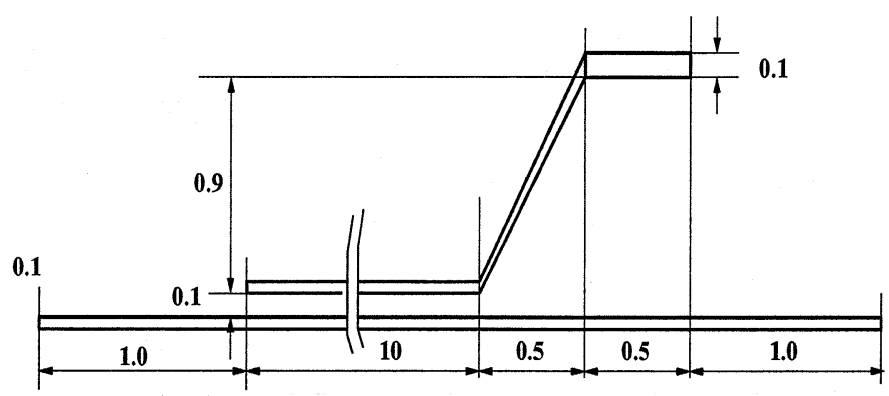

Fig. 11. Side view of differential connection.

case is also very close, within a percent of the theoretical resonance frequency of $750 \mathrm{MHz}$.

This second example, a TD skin effect TL example shown in Fig. 7, is designed to give a comparison with an accurate Spice-type solution. This example also illustrates that accurate 


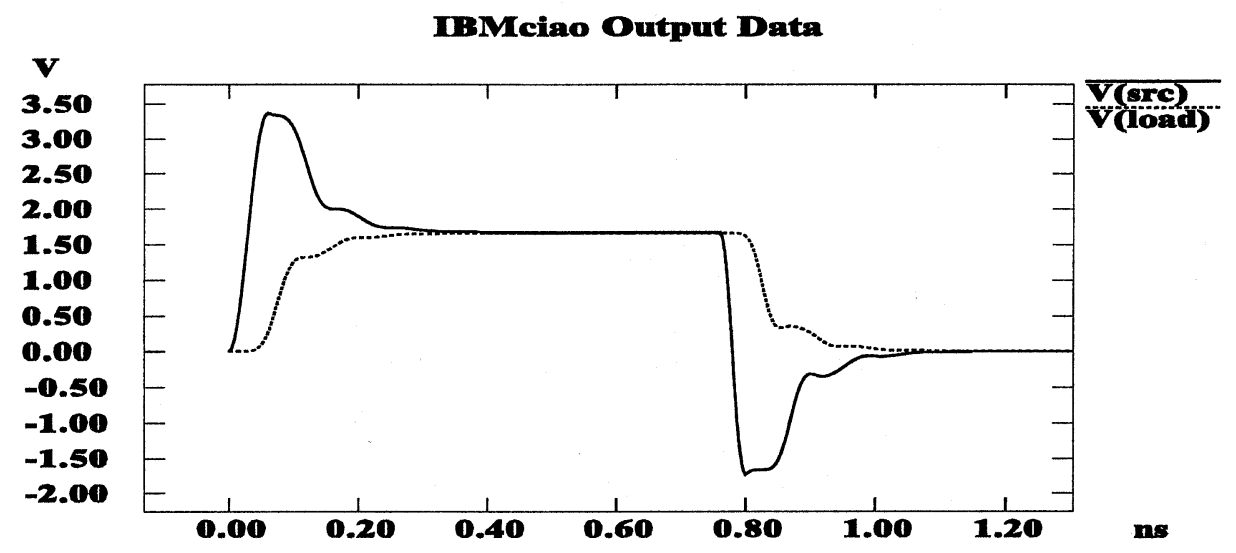

Fig. 12. Analysis results for structure in Fig. 10.

answers can be obtained even for very large cell aspect ratios. In this case, the cell thickness to length ratio is as large as $1: 4750$. The cross section is subdivided for the volume filament (VFI) skin effect representation with one cell along the thickness and ten cells along the width. The length is subdivided into 20 nonuniform cells where the cell size is decreasing toward the ends. In this comparison, we used the PowerSpice circuit solver which uses a method of characteristics based TL formulation [?]. Fig. 8 shows that the three-dimensional (3-D) PEEC model and the two-dimensional (2-D) TL Spice solver give very close voltage waveforms at the beginning and the end of the line. The new nonorthogonal formulation increases the number of electrical structures that can be analyzed using PEEC. Examples of nonorthogonal problems are wire bonds, diagonal or PC board interconnects, and chip interconnects which are at an arbitrary angle. Unfortunately, very few well-documented results exist in this class of problems today. The volumetric hexahedral meshing for nonorthogonal structures is very complex. Most modern software toolkits available for meshing are designed for mechanical or hydraulic analysis where the mesh structures is based on hexahedra. Our hexahedral meshing is an extension of the original rectangular approach [36]. It is node based as is shown in Fig. 2 for a zero thickness structure. Four different sub-meshes are associated with this node based mesh, a quadrilateral surface mesh for the capacitive cells, and three hexadral volumetric meshes for the inductive cells in the local $\hat{\boldsymbol{a}}, \hat{\boldsymbol{b}}$, and $\hat{\boldsymbol{c}}$ directions. To create these meshes, we use the local coordinates as described in Section II-A. Fig. 9 illustrates this for a hexahedral block which is subdivided into two inductive cells for the front directed $\hat{\boldsymbol{a}}$, and one along the right directed $\hat{\boldsymbol{b}}$ and one along the top direction $\hat{\boldsymbol{c}}$. Hence, there are three corner nodes in the $\hat{\boldsymbol{a}}$ direction, and two corner nodes in the $\hat{\boldsymbol{b}}$ and $\hat{\boldsymbol{c}}$ directions.

Computationally, the fast $O\left(N^{2}\right)$ TD approach [14], [58] is always preferable and faster where applicable than the $O\left(N^{3}\right)$ frequency-domain techniques. However, in the nonorthogonal case the runtime and memory usage of the analysis is impacted by the presence of the nonorthogonal elements due to the more complex element computations in the new formulation. In the orthogonal code, inductive coupling is confined to the currents in the same direction. This is no longer the case for arbitrary oriented conductors and more inductive cells will be coupled. As a test of the nonorthogonal code, we modeled a short inter- connect structure between a drive chip and a $50-\Omega$ resistive load shown in Fig. 10. The interconnect example consists of two lines above a ground plane with a nonorthogonal copper connection part embedded in air, or $\epsilon_{r}=1$. The ground plane and the wires are $0.05-\mathrm{mm}$ thick with the exception of the upper connections which are $0.1-\mathrm{mm}$ thick. The width of all wires is $0.1 \mathrm{~mm}$ with a 0.2-mm spacing while more geometrical dimensions are shown Fig. 11. The only part not shown in this figure is the bend which starts at $6.0 \mathrm{~mm}$ and ends at $8.0 \mathrm{~mm}$, measured at the two corners where the wire bends. This is shown in Fig. 10. The spacing between the wires in the bend section is increased to $0.5 \mathrm{~mm}$. The two lines are driven differentially, by two current source pulses of the same magnitude and opposite directions in parallel with a $50-\Omega$ resistor. The sources have a common centered ground connected to the plane. The input waveform is a sine square wave shape with a rise time of $60 \mathrm{ps}$, a fall time of $40 \mathrm{ps}$ and a pulse width of 0.7 ns. Fig. 12 shows the results of a TD analysis for the example in Fig. 10. The differential voltage-time waveforms are shown at the source as well as at the load. To approximately verify the solution, we used a Manhattan (rectangular) approximation of the geometry and the waveforms were slightly different as expected.

\section{CONCLUSION}

The formulation given in this paper extends the PEEC approach in a consistent way to general nonorthogonal geometries. Importantly, the approach converges to the rectangular formulation for orthogonal geometries. Further, like the original PEEC formulation it includes the VFI model for the skin effect and a model for dielectric blocks. The examples in the paper show some of the versatility of the circuit oriented method. It is also shown that the ability to analyze in the TD and frequency domain, including other circuit elements leads to the best possible results for each problem at hand.

\section{REFERENCES}

[1] B. Archambeault, O. Ramahi, and C. Brench, EMI/EMC Computational Modeling Handbook. Norwell, MA: Kluwer, 1998.

[2] C. Christopoulos, An Introduction to the Transmission-Line Modeling TLM Method. New York: IEEE Press, 1992.

[3] W. Sui, Time-Domain Computer Analysis of Nonlinear Hybrid Systems. Boca Raton, FL: CRC, 2002. 
[4] A. E. Ruehli and P. A. Brennan, "Efficient capacitance calculations for three-dimensional multiconductor systems," IEEE Trans. Microwave Theory Tech., vol. MTT-21, pp. 76-82, Feb. 1973.

[5] K. Nabors and J. White, "FastCap: A multipole accelerated 3-D capacitance extraction program," IEEE Trans. Computer-Aided Design, vol. 10, pp. 1447-1459, Nov. 1991

[6] Y. L. Le Coz and R. B. Iverson, "A stochastic algorithm for high-speed capacitance extraction in integrated circuits," IEEE Trans. Microwave Theory Tech., vol. 40, pp. 1507-1516, July 1992.

[7] A. E. Ruehli, "Inductance calculations in a complex integrated circuit environment," IBM J. Res. Develop., vol. 16, no. 5, pp. 470-481, 1972.

[8] P. A. Brennan, N. Raver, and A. E. Ruehli, "Three-dimensional inductance computations with partial element equivalent circuits," IBM J. Res. Develop., vol. 23, no. 6, pp. 661-668, 1979.

[9] P. K. Wolff and A. E. Ruehli, "Inductance computations for complex three dimensional geometries," in Proc. IEEE Int. Symp. Circuits and Systems, vol. 2, 1981, pp. 16-19.

[10] C. Paul, Analysis of Multiconductor Transmission Lines. New York: Wiley, 1992

[11] R.-B. Wu, C.-N. Kuo, and K. K. Chang, "Inductance and resistance computations for three-dimensional multiconductor interconnection structures," IEEE Trans. Microwave Theory Tech., vol. 40, pp. 263-270, Feb. 1992.

[12] M. Kamon, M. J. Tsuk, and J. White, "Fasthenry: A multipole-accelerated 3-D inductance extraction program," in Proc. Design Automation Conf., vol. 1, June 1993, pp. 678-683.

[13] A. E. Ruehli and H. Heeb, "Circuit models for three-dimensional geometries including dielectrics," IEEE Trans. Microwave Theory Tech., vol. 40, pp. 1507-1516, July 1992.

[14] W. Pinello, A. C. Cangellaris, and A. E. Ruehli, "Hybrid electromagnetic modeling of noise interactions in packaged electronics based on the partial-element equivalent circuit formulation," IEEE Trans. Microwave Theory Tech., vol. 45, pp. 1889-1896, Oct. 1997.

[15] G. Antonini, S. Cristina, and A. Orlandi, "PEEC modeling of highvoltage tower under direct and nearby lightning strike," in Proc. 10th Int. Symp. High Voltage Eng. ISH, vol. 10, Montreal, CA, Aug. 1997.

[16] H. Shi, J. Fan, and J. Drewniak, "Modeling multilayered PCB power-bus designs using an MPIE based circuit extraction technique," in Proc. IEEE Int. Symp. Electromagnetic Compatibility, vol. 2, Denver, CO, Aug. 1998, pp. 647-651.

[17] N. Marques, M. Kamon, J. White, and M. Silveira, "A mixednodal-mesh formulation for efficient extraction and passive reduced-order modeling of 3-D interconnects," in Proc. Design Automation Conf., vol. 35, San Francisco, CA, 1998, pp. 297-302.

[18] G. Antonini, S. Cristina, and A. Orlandi, "PEEC modeling of lightning protection system and coupling to coaxial cables," IEEE Trans. Electromagn. Compat., vol. 40, pp. 481-491, Nov. 1998

[19] A. M. Nikenejad and R. G. Meyer, "Analysis, design, and optimization of spiral inductors and transformers for SI RF ICs," IEEE J. Solid-State Circuits, vol. 33, pp. 1470-1481, Oct. 1998.

[20] G. Wollenberg and A. Görisch, "Analysis of 3-D interconnect structures with PEEC using SPICE," IEEE Trans. Electromagn. Compat., vol. 41, pp. 412-417, Nov. 1999.

[21] M. Tröscher and H. Katzier, "Efficient PEEC modeling of multi-layer boards and multi-chip modules," in Proc. Int. Zurich Symp. EMC, vol. 14, Zürich, Switzerland, Feb. 2001, pp. 269-273.

[22] J. F. Fan, H. Shi, A. Orlandi, J. L. Knighten, and J. L. Drewniak, "Modeling DC power-bus structures with vertical discontinuities using a circuit extraction approach based on a mixed-potential intergral equation formulation," IEEE Trans. Adv. Packag., vol. 24, pp. 143-157, May 2001.

[23] R. F. Milsom, K. J. Scott, G. Clark, J. C. McEntegart, S. Ahmed, and F. N. Soper, "FACET-A CAE system for RF analogue simulation including layout," in Proc. Design Automation Conf., vol. 26, 1989, pp. 622-625.

[24] Y. Wang, V. Jandjayla, and C.-J. R. Shi, "Coupled electromagnetic-circuit simulation of arbitrary-shaped conducting structures," in Dig. Electr. Perf. Electronic Packaging Conf., vol. 10, Boston, MA, Oct. 2001, pp. 233-236.

[25] A. Rong and A. C. Cangellaris, "Generalized PEEC models for three-dimensional interconnect structures and integrated passives of arbitrary shapes," in Dig. Electr. Perf. Electronic Packaging Conf., vol. 10, Boston, MA, Oct. 2001, pp. 225-228.

[26] A. E. Ruehli, G. Antonini, and A. Orlandi, "Extension of the partial element equivalent circuit method to non rectangular geometries," in Proc. IEEE Int. Symp. Electromagnetic Compatibility, Seattle, WA, Aug. 1999, pp. 728-733.
[27] G. Antonini, A. Ruehli, and J. Esch, "Nonorthogonal PEEC formulation for time and frequency-domain modeling," in Proc. IEEE Int. Symp. Electromagnetic Compatibility, vol. 1, Minneapolis, MN, Aug. 2002.

[28] B. M. Kolundzija and B. D. Popovic, "Entire-domain Galerkin method for analysis of metallic antennas and scatterers," Proc. Inst. Elect. Eng. $H$, vol. 140, no. 1, pp. 1-10, 1993.

[29] H. Singer, H.-D. Brüns, and G. Burger, "State of the art in the moment method," in Proc. IEEE Int. Symp. Electromagnetic Compatibility, Santa Clara, CA, Aug. 1996, pp. 122-227.

[30] A. W. Glisson and D. R. Wilson, "Simple and efficient numerical methods for problems of electromagnetic radiation and scattering from surfaces," IEEE Trans. Antennas Propagat., vol. AP-28, pp. 593-603, 1980.

[31] A. E. Ruehli, J. Garrett, and C. R. Paul, "Circuit models for 3-D structures with incident fields," in Proc. IEEE Int. Symp. Electromagnetic Compatibility, Dallas, TX, Aug. 1993, pp. 28-31.

[32] A. E. Ruehli, G. Papadopoulos, K. Aygün, E. Michielssen, and A. Cangellaris, "Transient analysis for printed circuit board problem using two different solvers," in Proc. IEEE Int. Symp. Electromagnetic Compatibility, Montreal, QC, Canada, Aug. 2001, pp. 1327-1332.

[33] B. Archambeault and A. E. Ruehli, "Analysis of power/ground-plane EMI decoupling performance using the partial-element equivalent circuit technique," IEEE Trans. Electromagn. Compat., vol. 43, pp. 437-445, Nov. 2001

[34] C.-T. Tai, Generalized Vector and Dyadic Analysis. New York: IEEE Press, 1992.

[35] C. A. Balanis, Advanced Engineering Electromagnetics. New York: Wiley, 1989.

[36] A. E. Ruehli, "Equivalent circuit models for three dimensional multiconductor systems," IEEE Trans. Microwave Theory Tech., vol. MTT-22, pp. 216-221, Mar. 1974.

[37] G. Antonini, A. Orlandi, and A. E. Ruehli, "Speed-up of PEEC method by using wavelet transform," in Proc. IEEE Int. Symp. Electromagnetic Compatibility, Washington, DC, Aug. 2000.

[38] A. E. Ruehli and H. Heeb, "Circuit models for three-dimensional geometries including dielectrics," IEEE Trans. Microwave Theory Tech., vol. 40, pp. 1507-1516, July 1992.

[39] S. Ramo, J. R. Whinnery, and T. Van Duzer, Fields and Waves in Communication Electronics. New York: Wiley, 1994.

[40] C. Ho, A. Ruehli, and P. Brennan, "The modified nodal approach to network analysis," IEEE Trans. Circuits Syst., vol. CAS-32, pp. 504-509, June 1975.

[41] J. Garrett, A. E. Ruehli, and C. R. Paul, "Accuracy and stability improvements of integral equation models using the partial element equivalent circuit PEEC approach," IEEE Trans. Antennas Propagat., vol. 46, pp. 1824-1831, Dec. 1998.

[42] F. W. Grover, Inductance Calculations: Working Formulas and Tables. New York: Dover, 1962.

[43] T.-H. Chen, C. Luk, H. Kim, and C. C. P. Chen, "Inductwise: Inductance-wise interconnect simulator and extractor," in Proc. IEEE Int. Conf. Computer-Aided Design, San Jose, CA, Nov. 2002, pp. 215-220.

[44] J.-T. Kuo and K.-Y. Su, "Analytical evaluation of the MoM elements for the capacitance of a charged plate," IEEE Trans. Microwave Theory Tech., vol. 50, pp. 1435-1436, May 2002.

[45] G. Antonini, A. Orlandi, and A. E. Ruehli, "Analytical integration of quasistatic potential intergrals on nonorghogonal coplanar quadrilaterals for the PEEC method," IEEE Trans. Electromagn. Compat., vol. 44, pp. 399-403, May 2002.

[46] D. A. Lindholm, "Three-dimensional magnetostatic fields from point matching integral equations with linearly varying scalar sources," IEEE Trans. Magn., vol. MAG-20, pp. 2025-2032, Sept. 1984.

[47] A. E. Ruehli, Ed., Circuit Analysis, Simulation and Design, Part 2. Amsterdam, The Netherlands: Elsevier Science, 1987.

[48] J. Vlach and K. Singhal, Computer Methods for Circuit Simulation. New York: Van Nostrand Reinhold, 1983.

[49] L. T. Pillage, R. A. Rohrer, and C. Visweswariah, Electronic Circuits and Systems Simulation Methods. New York: McGraw-Hill, 1995.

[50] A. E. Ruehli, N. B. Rabbat, and H. Y. Hsieh, "Macromodular latent solutions of digital networks including interconnects," in Proc. IEEE Int. Symp. Circuits and Systems, vol. 1, May 1978, pp. 515-512.

[51] H. Heeb, S. Ponnapalli, and A. E. Ruehli, "Frequency-domain microwave modeling using retarded partial element equivalent circuits," in Proc. Design Automation Conf., Dallas, TX, June 1993, pp. 702-706.

[52] J. E. Garrett, A. E. Ruehli, and C. R. Paul, "Efficient frequency-domain solutions for sPEEC EFIE for modeling 3-D geom," in Proc. Int. Zurich Symp. EMC, vol. 10, Zürich, Switzerland, Mar. 1995, pp. 179-184. 
[53] E. Chiprout and M. S. Nakhla, Asymptotic Waveform Evaluation. Norwell, MA: Kluwer, 1994.

[54] B. P. Rynne, "Comments on a stable procedure in calculating the transient scattering by conducting surfaces of arbitrary shape," IEEE Trans. Antennas Propagat., vol. 41, pp. 517-520, Apr. 1993.

[55] K. Aygün, B. Shanker, A. Ergin, and E. Michielssen, "A two-level plane wave time-domain algorithm for fast analysis of EMC/EMI problems," IEEE Trans. Electromagn. Compat., vol. 44, pp. 152-163, Feb. 2002

[56] S. M. Rao and T. K. Sarkar, "Transient analysis of electromagnetic scattering from wire structures using an implicit time-domain integral equation technique," Microwave Opt. Technol. Lett., vol. 17, no. 1, pp. 66-69, 1998.

[57] M. J. Bluck and S. P. Walker, "Time-domain analysis of large three-dimensional electromagnetic scattering problems," IEEE Trans. Antennas Propagat., vol. 45, pp. 894-901, May 1997.

[58] P. Restle, A. E. Ruehli, S. G. Walker, and G. Papadopoulos, "Full-wave peec time-domain method for the modeling of on-chip interconnects," IEEE Trans. Computer-Aided Design, vol. 20, pp. 877-887, July 2001.

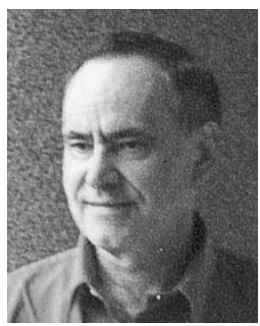

Albert E. Ruehli (M'65-M'74-F'84-LF'02) received the Ph.D. degree in electrical engineering from the University of Vermont, Burlington, in 1972.

$\mathrm{He}$ has been a member of various projects with IBM including mathematical analysis, semiconductor circuits and devices modeling, and as manager of a VLSI design and CAD group. Since 1972, he has been at the IBM T. J. Watson Research Center, Yorktown Heights, NY, where he now is a Research Staff Member in the Electromagnetic Analysis Group. He is the Editor of two books, Circuit Analysis, Simulation and Design (New York: North Holland, 1986, 1987 ) and author or coauthor of over 100 technical papers.

Dr. Ruehli has served in numerous capacities for the IEEE. In 1984 and 1985, he was Technical and General Chairman, respectively, of the ICCD International Conference. He has been a member of the IEEE ADCOM for the Circuit and System Society and an Associate Editor for the TRANSACTIONS ON COMPUTER-AIDED DESIGN. He has given talks at universities including keynote addresses and tutorials at conferences, and has organized many sessions. $\mathrm{He}$ received IBM Research Division or IBM Outstanding Contribution Awards in 1975, 1978, 1982, 1995, and 2000. In 1982, he received the Guillemin-Cauer Prize Award for his work on waveform relaxation, and in 1999, the Golden Jubilee Medal, both from the IEEE CAS Society. In 2001, he received a Certificate of Achievement from the IEEE EMC Society for inductance concepts and the partial element equivalent circuit method. He is a member of SIAM.

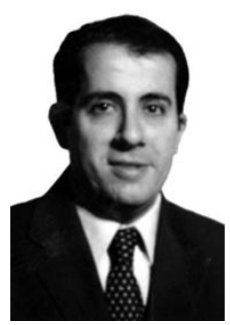

Giulio Antonini (M'94) received the Laurea degree (summa cum laude) in electrical engineering from the University of L'Aquila, L'Aquila, Italy, in 1994, and the Ph.D. degree in electrical engineering from the University of Rome "La Sapienza", La Sapienza, Italy, in 1998.

Since 1998, he has been with the UAq EMC Laboratory, Department of Electrical Engineering, University of L'Aquila, where he is a tenured Assistant Professor. His research interests focus on EMC analysis, numerical modeling, and signal integrity for high-speed digital systems. Since 1998, he collaborates with the IBM T. J. Watson Research Center, Yorktown Heights, NY, in the developments of algorithms for partial element equivalent circuit modeling.

In 1998, he received the Best Transactions Paper Award for a paper published in the IEEE TRANSACTIONS ON ElECTROMAGNETIC COMPATIBILITY, in 1997. Dr. Antonini is a member of the IEEE EMC TC-9 Committee.
Joris Esch received the B.S. degree in engineering and M.S. degree in mechanical and electrical engineering from the Katholieke Universiteit Leuven, Leuven, Belgium, in 1997 and 2000, respectively. He is currently working toward the $\mathrm{Ph}$.D. degree in electrical engineering at the Integrated Systems Laboratory, Columbia University, New York City.

From 2000 through 2002, he worked in an electromagnetic software development group at the IBM Thomas J. Watson Research Center, Yorktown Heights, NY. His research interests include numerical methods, circuit analysis and electromagnetic simulation.

Jonas Ekman was born in Boden, Sweden, on September 26, 1972. He is currently working toward the Ph.D. degree in electrical engineering at Luleå University of Technology, Sweden.

His field of study is computational electromagnetics, and, in particular, the use of the partial element equivalent circuit method for realistic electromagnetic modeling. During 2001 and 2002, he was a visiting Ph.D. student at the EMC Laboratory, University of L'Aquila, L'Aquila, Italy, and at IBM Thomas J. Watson Research Center, Yorktown Heights, NY.

Anita Mayo received the Ph.D. degree in mathematics from New York University, New York, in 1979.

She then taught at the University of California at Berkely, Stanford University, Stanford, CA, and the State University of New York, Stony Brook, before joining the research staff at the IBM Thomas J. Watson Research Center, Yorktown Heights, NY, in 1984. She has developed efficient numerical methods for solving a variety of differential equations on general regions. While at IBM, she also worked on problems arising in the manufacture of IBM products. Specifically, she was involved in developing and implementing methods that were used in magnetic calculations for designing recording heads, and for solving problems in electrodeposition, printing and packaging. Her most current area of work is computational finance.

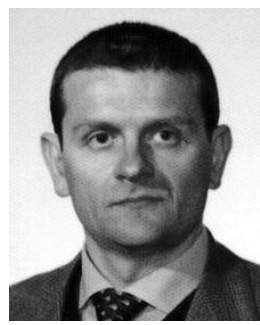

Antonio Orlandi (M'90-SM'97) was born in Milan, Italy, in 1963. He received the Laurea degree in electrical engineering from the University of Rome "La Sapienza," Italy, in 1988.

He was with the Department of Electrical Engineering, University of Rome "La Sapienza," from 1988 to 1990. Since 1990, he has been with the Department of Electrical Engineering of the University of L'Aquila. L'Aquila, Italy, where he is currently Full Professor. The author of more than 100 technical papers, he has published in the field of electromagnetic compatibility in lightning protection systems and power drive systems. Current research interests are in the field of numerical methods and modeling techniques to approach signal integrity, EMC/EMI issues in high-speed digital systems.

Dr. Orlandi received the IEEE TRANSACTIONS ON ELECTROMAGNETIC Compatibility Best Paper Award in 1997. He is Member of the Education, TC-9 Computational Electromagnetics and TC-10 Signal Integrity Committees of the IEEE EMC Society, Chairman of the "EMC INNOVATION" Technical Committee of the International Zurich Symposium and Technical Exhibition on EMC. From 1996 to 2000, has been Associate Editor of the IEEE TRANSACTIONS ON ELECTROMAGNETIC COMPATIBILITY and now serves as Associate Editor of the IEEE TRANSACTIONS ON MOBILE COMPUTING. 